\title{
Comparative Study of Image Thresholding Using Type-2 Fuzzy Sets and Cloud Model
}

\author{
Tao Wu ${ }^{*}$ \\ State Key Laboratory of Software Engineering, Wuhan University \\ Wuhan, Hubei 430079, China \\ School of Information Science and Technology, Zhanjiang Normal University \\ Zhanjiang, Guangdong 524048, China \\ Kun Qin \\ School of Remote Sensing and Information Engineering, Wuhan University \\ Wuhan, Hubei 430079, China \\ E-mail: qinkun163@163.com
}

\begin{abstract}
Uncertainty is an inherent part of image segmentation in real world applications. The use of new methods for handling incomplete information is of fundamental importance. Type-1 fuzzy sets used in conventional image segmentation cannot fully handle the uncertainties. Type-2 fuzzy sets and cloud model can handle such uncertainties in a better way because they provide us with more design degrees of freedom. The paper presents a comparison on the two approaches for image segmentation with uncertainty, that is, image thresholding based on type-2 fuzzy sets and cloud model. Firstly, the theoretical foundations of two methods are analyzed. Secondly, the processing of image segmentation with uncertainty is compared through two stages respectively, which is histogram analysis and optimum threshold selection. Finally, the experiments are divided in three groups, both synthetic and real images are used to investigate the performance of handling uncertainty in image segmentation, and some noisy images are also involved in to validate the performance of suppressing noise. The experimental results suggest that the conclusion of comparisons is effective.
\end{abstract}

Keywords: Type-2 fuzzy sets; cloud model; image thresholding; image segmentation; uncertainty.

\section{Introduction}

Image segmentation is often described as the process that subdivides an image into its constituent parts and extracts those parts of interest objects, and it is the first step and also one of the most critical tasks of automatic image analysis ${ }^{1,2}$. In spite of several decades of investigation, image segmentation is still a challenging research topic. Extensive research has been done in creating many different approaches and algorithms for image segmentation, in which image thresholding is a most simple and effective method ${ }^{3-5}$. There are several uncertainties in image thresholding, including grayness ambiguities, imprecision, instabilities, and with noises.

*Corresponding author, Address: State Key Laboratory of Software Engineering, Wuhan University, Luojia Hill, Hubei, Wuhan 430072; E-mail: taowu0706@gmail.com. 
It became an important topic that how to introduce uncertainty theories and methods to represent, evaluate, or remove these uncertainties in a robust way ${ }^{6-8}$.

The theoretical foundation of fuzzy techniques is fuzzy sets proposed by Zadeh in $1965^{9}$, which uses precise membership or membership function to measure the fuzziness, and it is suitable to develop the new algorithms for image segmentation with uncertainty ${ }^{10}$. Considering the uncertainty of membership grade, Zadeh proposed type-2 fuzzy sets ${ }^{11}$, Mendel develop the concept and provide further researches ${ }^{12}$. Similarly, Li proposed cloud model in $1995^{13-15}$, which automatically produces membership grade based on probability measure space. The two methods both concentrate on the essentials of uncertainty and have been applied in many fields for more than ten years ${ }^{12,13,16}$.

It is difficult to choose the membership function for traditional fuzzy sets, and the current researches of fuzzy image thresholding focus on the applications of extensions of fuzzy sets ${ }^{10,17}$, in which an image thresholding algorithm using interval type-2 fuzzy set (ITT2) proposed by Tizhoosh is as a modern standard $^{18,19}$. Some comparative studies have indicated that the contribution based on type- 2 fuzzy set is important ${ }^{17,20}$.

But the fuzzy sets based methods only consider the fuzziness on image thresholding. In fact, there is a strong association between fuzziness and randomness. On the one hand, the membership grade indicates randomness with the different conditions, on the other hand, a higher probability means a greater membership grade, and then it is a key problem that how to introduce probability measure space into the representation of membership grade and establish the relationships between fuzziness and randomness ${ }^{13,15}$.

Based on one-dimensional cloud model, an image thresholding method (ITC) is proposed using onedimensional gray histogram analysis ${ }^{21}$. After that, Qin et al analyze the root of uncertainty in image segmentation, then improve and develop the cloud model based method $^{22,23}$.

Comparative with traditional methods for image segmentation, these new methods (i.e. ITT2 and ITC) can capture uncertainty and produce good results when the lack of knowledge of the expert, because the new methods introduce the theories, type-2 fuzzy sets or cloud model, into image segmentation, and analyze the histogram with uncertainty. But even so, the theory fundament of the two methods is quite different. This paper provides the comparative studies between them to illustrate their advantages and disadvantages, their connections and differences, and make a reference for the novel image segmentation with uncertainty. Meanwhile, we show some experimental results to support our views.

The rest of the paper is organized as following. Section 2 presents an introductory explanation of type- 2 fuzzy sets and cloud model, and provides comparative analysis on the theory basic of the selected methods. And then, Section 3 is an overview of the selected image thresholding methods involved in the experimental comparisons, and remarks on their variations for histogram analysis and thresholding selection in detail. Next, Section 4 shows the experimental results. Finally, the discussions and conclusion of the experiments is drawn in Section 5.

\section{Comparison on the theory basic of selected methods}

\subsection{Interval type-2 fuzzy sets}

Although the motivation of ITT2 proposed by Tizhoosh is to remove the uncertainty of membership values by using type-2 fuzzy sets, the proposed algorithm only used interval type-2 fuzzy sets, which are a particular case of type- 2 fuzzy sets. So in fact, the theory basic of ITT2 is interval type- 2 fuzzy sets.

A type- 2 fuzzy set, $\operatorname{denoted} \widetilde{A}$, is characterized by a type-2 membership function $\mu_{\tilde{A}}(x, u)$, where $x$, the primary variable, has domain $X$, and $u$, the secondary variable, has domain $u \in J_{x} \subseteq[0,1]$ at each $x \in X$, i. $\mathrm{e}^{24}$.

$$
\tilde{A}=\left\{\left((x, u), \mu_{\tilde{A}}(x, u)\right) \mid \forall x \in X, \forall u \in J_{x}\right\}
$$

where $0 \leq \mu_{\tilde{A}}(x, u) \leq 1$.

The membership grades of type- 2 fuzzy sets themselves are type- 1 fuzzy sets, which are denoted by a type-1 membership function, called secondary membership function. If the secondary membership 
functions are type- 1 interval sets, the type- 2 fuzzy set is called an interval type-2 fuzzy set. Interval type-2 fuzzy set is defined by lower membership function (LMF) and upper membership function (UMF). The region bounded by LMF and UMF is called the footprint of uncertainty (FOU). Each value of the primary variable $x$ of interval type-2 fuzzy set is an interval ${ }^{12}$.

\subsection{Normal cloud model}

In theory, there are several forms of Cloud model ${ }^{25}$, but the normal cloud model is commonly used in practice ${ }^{26}$. The universalities of normal distribution and bell membership function are the theoretical foundation for the universality of normal cloud model ${ }^{14}$.

Let $U$ be a quantitative universal set described by precise numbers, and $C$ be the qualitative concept related to $U$, if there is a number $x \in U$, which is a random realization of the concept $C$ and $x$ satisfies $x \sim N\left(E x, E n^{2}\right)$ where $E n^{\prime} \sim N\left(E n, H e^{2}\right)$, and the certainty degree of $x$ on $C$ is

$$
\mu(x)=\mathrm{e}^{-\frac{\left(x-E_{x}\right)^{2}}{2\left(E_{n}\right)^{2}}}
$$

then the distribution of $x$ on $U$ is a normal cloud ${ }^{13}$.

The overall property of a concept can be represented by three numerical characters, Expected value $E x$, Entropy $E n$ and Hyper-entropy He. Ex is the mathematical expectation of the cloud drops. $E n$ is the uncertainty measurement of the qualitative concept, and determined by both the randomness and the fuzziness of the concept. $\mathrm{He}$ is the uncertain measurement of entropy $^{13}$.

The universality and mathematical properties of normal cloud has been proved, and more information about cloud, including forward normal cloud generator, backward cloud generator and cloud transformation, can be obtained from reference ${ }^{13}$.

\subsection{Comparisons on interval type-2 fuzzy sets and normal cloud model}

Interval type-2 fuzzy sets and normal cloud model are methods to analyze qualitative concept with uncertainty, but there are some differences between them ${ }^{16}$. Interval type-2 fuzzy sets represent the uncertainty of membership function through FOU determined by the mathematical functions of UMF and LMF, and use two mathematical functions to solve problems. Normal cloud model generates the membership grades using forward normal cloud generator, which is a random value with stabilization tendency. Normal cloud model uses probability and mathematical statistics to solve problems.

We take the interval type-2 fuzzy set with Gaussian primary membership function and normal cloud model for example. Fig.1 illustrates a qualitative concept expressed by the two methods. In Fig.1(a), the concept is expressed by an interval type-2 fuzzy set. The UMF and $L M F$ are defined as $L M F=\exp \left[-\frac{(x-\mu)^{2}}{2 \sigma_{1}^{2}}\right]$, $U M F=\exp \left[-\frac{(x-\mu)^{2}}{2 \sigma_{2}^{2}}\right], \mu=25, \sigma_{1}=2, \sigma_{2}=3$. Fig.1(b) illustrates the expression by normal cloud

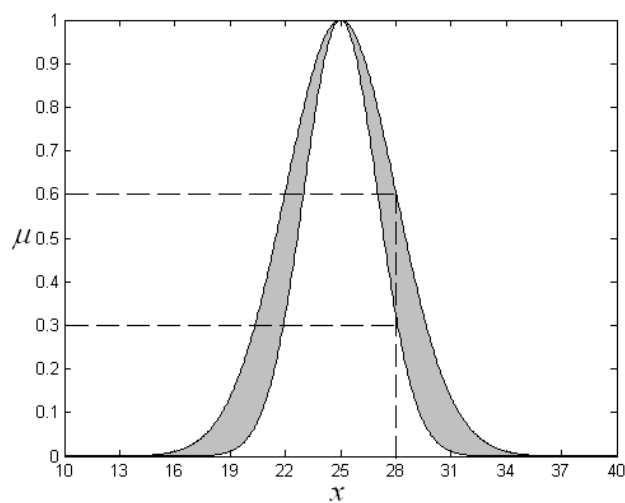

(a)

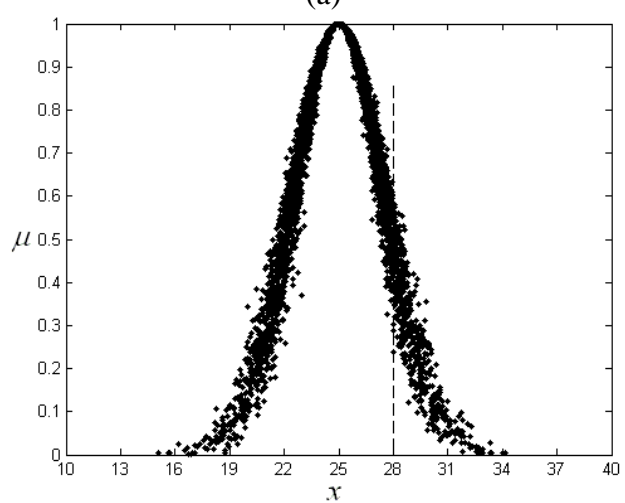

(b)

Fig. 1. Comparison on the theory basic of selected methods: (a) Interval type-2 fuzzy sets; (b) Normal loud model

model, $E x=25, E n=2.5, H e=0.25$. 
Interval type-2 fuzzy sets represent the uncertainty of qualitative concept through FOU. Normal cloud model represents qualitative concept through a model consisting of cloud drops. The overall shapes of both are similar but the expression forms are different. The FOU is consecutive, and has clear boundary. Normal cloud is composed of discrete points, and has no clear boundary.

Interval type-2 fuzzy sets study the fuzziness of membership grade, which is considered as second-order fuzziness. Normal loud model studies the randomness of membership grade, which is considered as fuzziness, randomness and the connection of them. Interval type-2 fuzzy sets take the membership grade as an interval. For example, as illustrated in Fig.1(a), the membership grade corresponding to $x=28$ is an interval [0.3,0.6], but the uncertainty of the interval is ignored. Interval type-2 fuzzy sets only capture first-order uncertainty. For normal cloud model, the membership grade is generated by forward normal cloud generator which is realized by program, when $x=28$, the membership grade is neither an exact value nor an interval, but a series of discrete points, as illustrated in Fig.1(b). Normal cloud model capture the randomness of the membership grade.

\section{Comparison on the selected methods}

\subsection{An overview of the selected methods}

$P=\{\langle x, y\rangle \in Z \times Z \mid x \in[1, w], y \in[1, h]\}$ is a finite set of pixels, an image is a pair $(P, I)$ consisting of $P$, and a mapping $I$ that assigns to each pixel $p=<x, y>\in P$ a pixel value $I(p) \in[0, L-1], h, w$ is height and width of the image, and $L$ is the gray level. hist $(g)$ with $g \in[0, L-1]$ denotes the histogram of the image.

ITT2 uses interval type-2 fuzzy set to create a mapping between pixel space and grayscale space. A new measure of ultrafuzziness as is introduced to quantify the vagueness of a type- 2 fuzzy set ${ }^{18}$.

$$
\gamma=\frac{1}{M N} \sum_{g=0}^{L-1} \operatorname{hist}(g) \times\left[\mu_{U}(g)-\mu_{L}(g)\right]
$$

where $\mu_{U}(g)=[\mu(g)]^{1 / \alpha}, \mu_{L}(g)=[\mu(g)]^{\alpha}, \alpha \in(1,2]$.

Shifting the membership function along the grayscale range, if the position $g_{o p t}$ is with maximum ultrafuzziness, $g_{\text {opt }}$ is as the optimal threshoding.
ITC uses cloud model to create a mapping between pixel space and grayscale space. The histogram of image is converted into a series of normal cloud models by cloud transformation.

$$
\operatorname{hist}(g) \rightarrow \sum_{i=1}^{n} a_{i} \times C\left(E x_{i}, E n_{i}, H e_{i}\right)
$$

where $a_{i}$ is a parameter representing the distribution frequency of a cloud model; $n$ represents the number of cloud model ${ }^{22}$.

A series of fundamental cloud models can be considered as the leaf nodes in a concept tree. Based on cloud synthesization, the two neighboring concepts are combined into one synthesized concept at a higher level, and then concepts with a satisfactory level (two cloud models corresponding to object or background respectively) are extracted in a bottom-up way, layer by layer. Each pixel is labeled according to the maximum certainty degree corresponding to object or background.

The two selected method both are image thresholding using grayscale histogram analysis, and the methods are rooted at the grayscale histogram of an image, which is used to approximate the unknown graylevel probability density function ${ }^{27}$. From the view of mathematical model, the methods create a mapping from pixel space to grayscale space, and search a suitable partition on the grayscale space, and then establish an inverse mapping from to grayscale space pixel space. The flow chart of the methods including five modules is as Fig.2, firstly, some possible parameters is initiated and grayscale histogram is calculated, secondly, histogram is analyzed using given models, and next optimal thresholding is obtained by related optimization methods, finally, the segmentation task can be performed. Although ITT2 and ITC are in the same framework, the theory fundament of the two methods is quite different. In the following, we compare

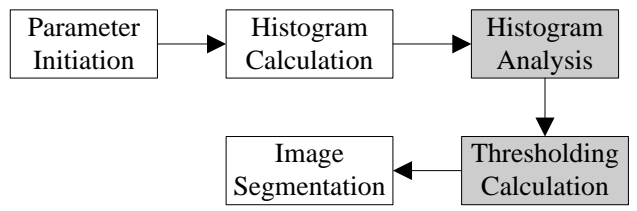

Fig. 2. The flow chart of image thresholding

the two methods from two modules, histogram analysis and thresholding calculation. 


\subsection{Comparison on the histogram analysis}

ITT2 defined the problem using clear mathematical function. After the shape of skeleton membership function $\mu(g)$ and parameter $\alpha$ are selected, the membership grade $\mu(g)$ of any gray level $g$ is extended as an interval $\left[[\mu(g)]^{\alpha},[\mu(g)]^{1 / \alpha}\right]$, and then an interval type-2 fuzzy set is obtained to approximate the grayscale histogram of an image. As is shown in Fig.3(a), ITT2 analyzes the histogram along the horizontal direction (grayscale), if the grayscale is 256 , then the method implicitly constructs 256 interval type2 fuzzy sets. In order to directly observe, Fig.3(a) only illustrates the principle using two type-2 fuzzy sets with grayscale $g=65$ or 180 , which are contiguous regions with a rectangular legend.

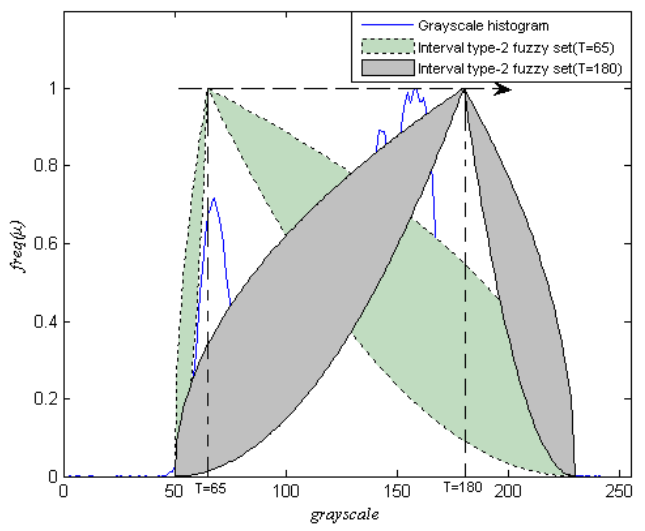

(a)

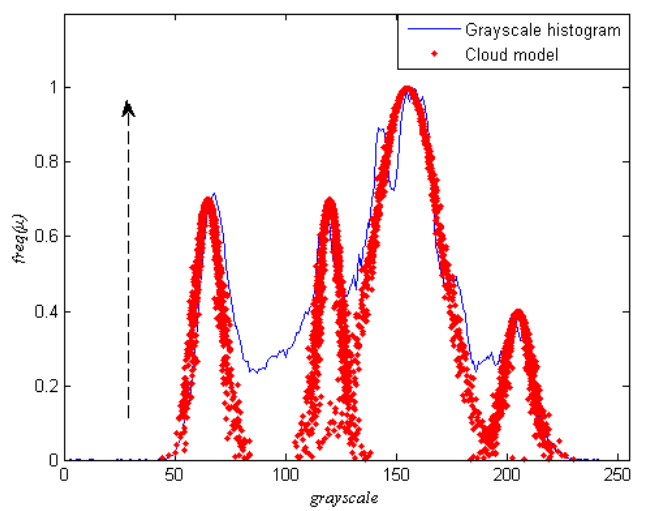

(b)

Fig. 3. Comparison on the histogram analysis: (a) ITT2; (b) ITC
Obviously, ITT2 is a supervised method that depends on the human, since the shape of skeleton membership function and parameter are the important prerequisite for successful segmentation. Assuming membership function and parameter is appropriate for the grayscale histogram, for example, the membership function reflects the outline of the histogram, the following steps will be performed smoothly, but not vice versa. In fact, it is still difficult that how to select the membership function and its parameter in practice.

ITC uses cloud concepts to analyze the histogram, and simulate the concepts forming process. In mathematics, every kind of probability distribution can be divided into the integration of some normal distributions. Similarly, considered the universality of normal cloud, the histogram can be looked as the integration of several normal clouds, and it carries out the conversion from quantity data to quality cloud concept.

The local maximum point is the convergence center of histogram and it is as the center of concept which represents the mathematics expectation of cloud model. The higher peak value indicates more data is converged, so these data will be prior to generate a quality concept, that is, a cloud model. The numerical section of the quality concept is minus from the original distribution, and then the next local maximum is found for another cloud model, until the parameter $\varepsilon$ is available, which reflects the magnitude of deviation between cloud models and the histogram. In Fig.3(b), it is an example of first 4 cloud models, which consist of non-contiguous points with a point legend. As can be seen from Fig.3(b), ITC analyzes the histogram along the vertical direction (frequency). In theory, more cloud models can be abstracted from the histogram, but if the parameter $\varepsilon$ is set effectively, the number of the cloud models is definite, and it is much less than the gray level.

Consequently, ITC is an unsupervised heuristic method without membership function and parameter. According to the frequency contribution of grayscale for the histogram, ITC extracts cloud models layer by layer, in which the sequence indicates the rank by contribution. There is only one parameter $\varepsilon$ in the process of extracting cloud models, and this parameter is easy to select and unnecessary to make a careful choice. 


\subsection{Comparison on the thresholding calculation}

Once an appropriate model for the histogram analysis is build, and the image is mapped from pixel space to grayscale space, the remaining problem is to determine at which grayscale the optimal thresholding is.

ITT2 searches the optimal thresholding with an exhaustive strategy. Along the grayscale, ITT2 tests all the candidate interval type-2 fuzzy sets one by one, as shown in Fig.4(a), the searching goal is to maximize the ultrafuzziness of interval type-2 fuzzy set. Essentially, ITT2 is a deterministic algorithm, which explicitly searches the optimal thresholding, any one execution of the algorithm produces the same result.

ITC is a probabilistic algorithm, rather than an exhaustive search. A series of fundamental cloud models are as the leaf nodes in a concept tree. Based on cloud synthesization, each pair of neighboring concepts is combined into one synthesized concept at a higher

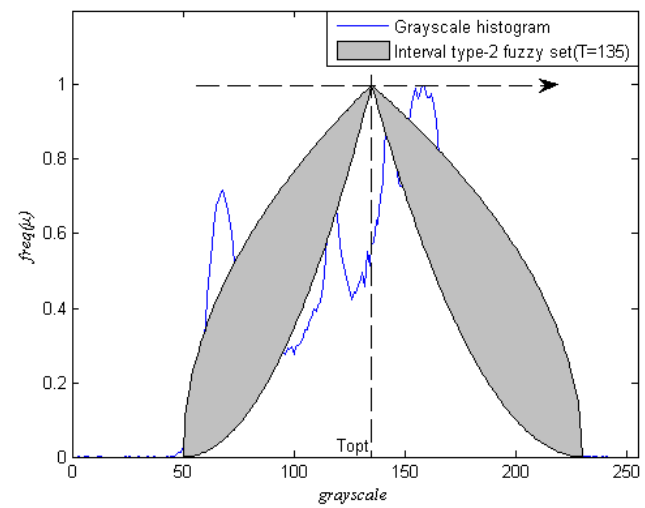

(a)

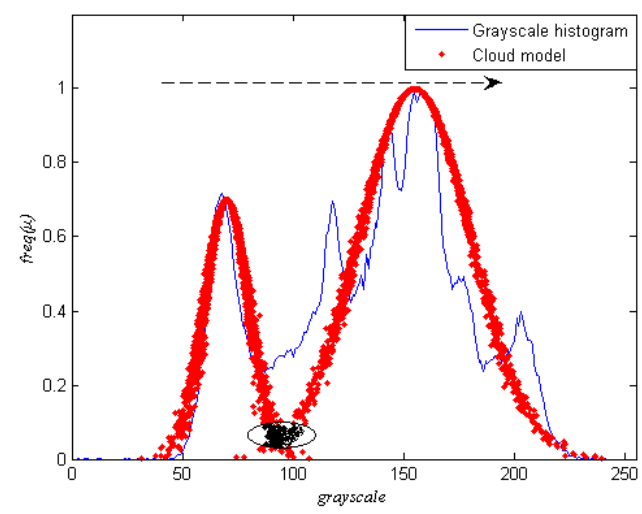

(b)

Fig. 4. Comparison on the optimal thresholding selection: (a) ITT2; (b) ITC level until only two cloud models remain which correspond to object or background respectively. One of cloud synthesization indicates that there is an agreement between the two cloud models in the current concept level. The goal of iterative synthesization is to discover two cloud models in the highest level to approximate the histogram, one denotes object, and the other denotes background. Each pixel of the image is labeled as object if the certainty degree belongs to object cloud model is greater than that of background. ITC uses soft segmentation rather than single thresholding. As is shown in Fig.4(b), there are overlaps between object cloud and background cloud, as the ellipse illustrated in Fig.4(b), whether or not the pixels corresponding to the grayscales of this section belong to object or background is uncertain. Essentially, ITC implicitly searches the optimal thresholding, any one execution of the algorithm maybe produce the different result, but the result is with a stable tendency.

\subsection{Comparison on the classification of methods}

With the algorithms having been developed, classification of techniques for image segmentation becomes essential. We compare the two selected methods from the classification of algorithms.

ITT2 is a kind of deductive method from generals to particulars, which uses a unified model (interval type-2 fuzzy set) to represent the histogram along the grayscale. Therefore, it is easy to structuring and forming with ITT2.

Compared with ITT2, ITC is a kind of inductive method from particulars to generals, which uses the method of probability and mathematical statistics, and heuristically extracts the cloud models from the histogram. So ITC is an unsupervised algorithm, which is more robust in theory.

\section{Experiments}

\subsection{Experimental setup}

To demonstrate the efficiency of the proposed comparisons, both synthetic and real images, with and without the contamination of Gaussian white noise and salt \& pepper noise, are used in the experiments. In the rest of this section, we conduct three groups of experiments to test the selected methods. Group one is 
designed to investigate the performances of the selected methods on the histogram analysis and optimal thresholding choice. Group two is designed to objectively compare the overall performances of the selected methods on image segmentation without noisy. Group three is designed to objectively compare the performances under noisy environment.

To compare the quality of the segmented images, we quantitatively evaluated the performance of the methods using misclassification error (ME). Considering image segmentation as a pixel classification process, the percentage of pixel misclassification is the discrepancy measure that comes most readily to human observation. ME reflects the percentage of background pixels wrongly assigned to foreground, and conversely, foreground pixels wrongly assigned to background. For the two-class segmentation problem, it can be expressed as $^{28}$

$$
M E=\left(1-\frac{\left|B_{o} \cap B_{t}\right|+\left|F_{o} \cap F_{t}\right|}{\left|B_{o}\right|+\left|B_{t}\right|}\right) \times 100 \%
$$

where background and foreground are denoted by $B_{o}$ and $F_{o}$ for the original image, and by $B_{t}$ and $F_{t}$ for the test image. $B_{o} \cap B_{t}$ is the number of background pixels rightly assigned to background, and $F_{o} \cap F_{t}$ vice versa.| | is the cardinality of a set. The ME varies from $0 \%$ for a perfectly classified image to $100 \%$ for a totally wrongly classified image.

To ensure the highest possible accuracy, the ground truth was created entirely by hand using the GNU image manipulation program (GIMP) ${ }^{29}$, and no semi-automatic technique was used. This was also important to avoid potential bias to any algorithmic facet of the procedure used to create it. But it should be noted that creating a $100 \%$ pixel accurate ground truth is, in general, impossible, due to the ambiguity in the true positions of the border pixels. ITT2 and ITC both are compiled in the MATLAB 2007b environment on a $2.31 \mathrm{GHz}$ Dual Core PC with 2.0GB RAM.

\subsection{Experimental results}

\subsubsection{Synthetic images}

It is usually desirable to test the thresholding algorithm using the synthetic images for which the ideal threshold can be identified directly ${ }^{30}$. To verify the comparisons on the histogram analysis and optimal thresholding selection, two synthetic images with $256 \times 256$ pixels are involved in three experiments, whose histograms are composed of mixtures with different statistical characteristics associated with the object and background classes, as shown in Fig.5. Fig.5(a) (circle) shows some round gray objects on the darker background, and ideal segmentation is shown in Fig.5(b). Fig.5(c) (square) shows some square gray objects on the darker background, the ideal segmentation is shown in Fig.5(d).

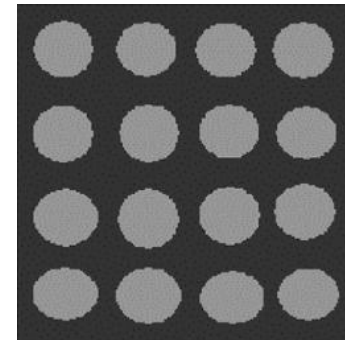

(a)

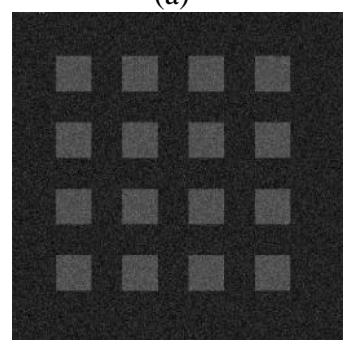

(c)

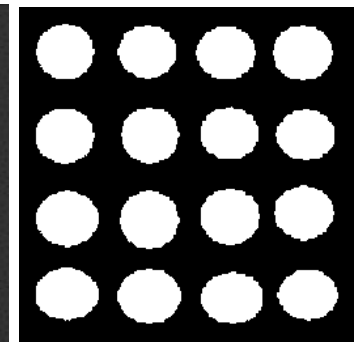

(b)

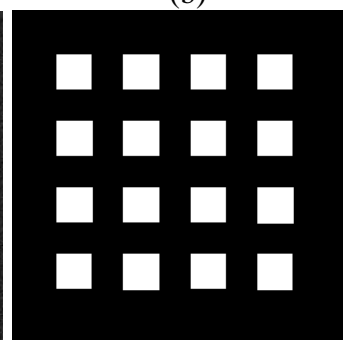

(d)
Fig. 5. Image and its ground-truth: (a) Circle; (b) Ground-truth of circle; (c) Square; (d) Ground-truth of square

Experiment 1: We conduct five independent experiments with various parameters. For ITT2, the skeleton membership function as

$$
\mu(g)=\left\{\begin{array}{cc}
0, \quad g \leq g_{\text {min }} & \text { or } \quad g \geq g_{\text {max }} \\
L(g)=\frac{g-g_{\text {min }}}{T-g_{\text {min }}}, & g_{\text {min }} \leq g \leq T \\
R(g)=\frac{g_{\text {max }}-g}{g_{\text {min }}-T}, & T \leq g \leq g_{\text {max }}
\end{array}\right.
$$

where parameter $\alpha$ is as $1.2,1.4,1.6,1.8$, and 2.0 respectively (labeled as $\mathrm{P}_{1}-\mathrm{P}_{5}$ ). For ITC, parameter $\varepsilon$ is as $0.01,0.002,0.001,0.0002$, and 0.0001. The ME values is calculated as (\%), and then the mean and standard variance is estimated by these five experiments. 
Tab. 1 and Tab. 2 show the experiment results. As can be seen from the tables, ITC with this group of parameters can be obtained better performances than ITT2. Although the parameters are varied too sharply (with a ratio 100:20:10:2:1), the increasing trends in ME values of ITC can be observed, but the change is not manifest. For the two images, the result of ITC obtains the minimal mean and standard deviation (STD) of ME values, so it indicates the smaller ratio of background pixels wrongly assigned to the foreground, and with the better stability. Conversely, the variation of parameters is much slightly (with a ratio 0.6:0.7:08:09:1), ITT2 fails to segment circle with stability, the ME values suddenly change from $1.1734 \%$ to $35.9528 \%$ when parameter $\alpha$ transformed between 1.6 and 1.8, what's more, this method fails to segment square under the five parameters, and the ME value is up to $19 \%$, obviously, ITT2 with this skeleton membership function is invalid to square. From Tab.1 and Tab.2, ITC is more robust than ITT2 in the first experiment.

Experiment 2: Considering ITT2 with the first skeleton membership function is useless, we design the second experiment to evaluate the performance with another skeleton membership function. The test images are the same as the first experiment, and the skeleton membership function is as

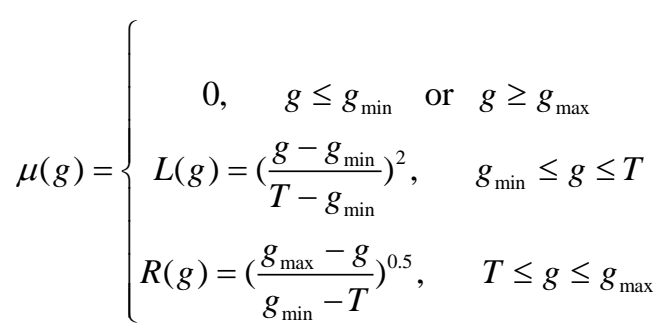

where parameter $\alpha$ is as 1.2, 1.6, 2.0, 3.0, and 4.0 respectively (labeled as $\mathrm{P}_{1}-\mathrm{P}_{5}$ ). As shown in Tab.3, the statistics of ME values is calculated similar to the first experiment. Compared Tab.2 and Tab.3, the second experiment obtains the better performance than the first one. But a traditional problem is arrived, for plenty of images, how to choose the skeleton membership function one by one is most difficult. Although the motivation of ITT2 is solve the problem of membership function selection in type-1 fuzzy sets, ITT2 extents the membership grade as an interval. Unfortunately, the new method is still involved in the old problem. In addition, Ref.18 noted that the parameter $\alpha \in(1,2]$ is usually meaningful for image data, but from Tab. 3, the experiment with $\alpha=4$ is still obtained good result under the skeleton membership function.

As a consequence of the above experiments, the minimal ME value denotes by bold and underline (ㅇ),

Table 1. Result of circle in experiment 1.

\begin{tabular}{lllllllll}
\hline Method & $\mathrm{P}_{1}$ & $\mathrm{P}_{2}$ & $\mathrm{P}_{3}$ & $\mathrm{P}_{4}$ & $\mathrm{P}_{5}$ & Parameter ratio & Mean of ME & STD of ME \\
\hline ITT2 & $\underline{\mathbf{3 5 . 9 5 2 8}}$ & 35.9528 & 35.9528 & $\mathbf{1 . 1 7 3 4}$ & 1.2115 & $0.6: 0.7: 0.8: 0.9: 1$ & 22.04866 & 19.03903 \\
ITC & $\underline{\mathbf{1 . 9 6 8 4}}$ & 1.9201 & 1.8082 & 1.0659 & $\mathbf{1 . 0 6 5 1}$ & $100: 20: 10: 2: 1$ & 1.56554 & 0.46016 \\
\hline
\end{tabular}

Table 2. Result of square in experiment 1.

\begin{tabular}{lllllllll}
\hline Method & $\mathrm{P}_{1}$ & $\mathrm{P}_{2}$ & $\mathrm{P}_{3}$ & $\mathrm{P}_{4}$ & $\mathrm{P}_{5}$ & Parameter ratio & Mean of ME & STD of ME \\
\hline ITT2 & $\underline{\mathbf{1 8 . 3 5 6 3}}$ & 18.3563 & 18.3563 & 18.3563 & 18.3563 & $0.6: 0.7: 0.8: 0.9: 1$ & 18.3563 & 0 \\
ITC & $\underline{\mathbf{9 . 3 5 3 5}}$ & 5.3488 & 0.6352 & 0.6828 & $\mathbf{0 . 5 6 9 0}$ & $100: 20: 10: 2: 1$ & 3.3178 & 3.9449 \\
\hline
\end{tabular}

Table 3. Result of of experiment 2 (ITT2).

\begin{tabular}{llllllll}
\hline Image & $\mathrm{P}_{1}$ & $\mathrm{P}_{2}$ & $\mathrm{P}_{3}$ & $\mathrm{P}_{4}$ & $\mathrm{P}_{5}$ & Mean of ME & STD of ME \\
\hline circle & 1.9302 & 1.9653 & 2.0645 & 2.2156 & 2.3407 & 2.10326 & 0.172832 \\
square & $\mathbf{0 . 5 9 0 6}$ & 0.6275 & 0.6275 & 0.8135 & 0.9750 & 0.72682 & 0.163853 \\
\hline
\end{tabular}

Table 4. Result of experiment 3 (ITC).

\begin{tabular}{|c|c|c|c|c|c|c|c|c|c|}
\hline$\overline{E_{1}}$ & $\mathrm{E}_{2}$ & $\mathrm{E}_{3}$ & $\mathrm{E}_{4}$ & $E_{5}$ & $\mathrm{E}_{6}$ & $\mathrm{E}_{7}$ & $\mathrm{E}_{8}$ & $\mathrm{E}_{9}$ & $\mathrm{E}_{10}$ \\
\hline 1.06506 & 1.06812 & 1.06354 & 1.06964 & 1.06506 & 1.05745 & 1.06964 & 1.06506 & 1.06812 & 1.07269 \\
\hline \multicolumn{5}{|c|}{ Mean of ME: 1.066438} & \multicolumn{5}{|c|}{ STD of ME: 0.00421809} \\
\hline
\end{tabular}


the maximal ME value denotes by bold and italic ( $\boldsymbol{0}$ ). Fig. 6 and Fig. 7 show the best segmentation and the worst segmentation obtained by ITT2 and ITC respectively.

For the image circle, ITT2 obtains the minimal ME value $1.1734 \%$ as shown in Fig.6(a), and the maximal ME value $35.9528 \%$ in Fig.6(c). ITC obtains the minimal ME value $1.0651 \%$ as shown in Fig.6(b), and the maximal ME value $1.9684 \%$ in Fig.6(d). For the best segmentation, the minimal ME value of ITT2 is less than ITC, but the difference is not enough for $0.1 \%$, which does not fall under human sight or observation. On the contrary, the maximal ME value of ITC is less than ITT2 for the worst segmentation, and the difference is up to $24 \%$, which leads to an obvious contrast, ITC produces an acceptable classified image, while ITT2 provides a wrongly classified image, and almost all the object pixels are assigned to background. In theory, a better classified image can be obtained by ITC if improving the parameter $\varepsilon$, but in contrast, the skeleton membership function and its parameter in ITT2 seem more difficult.

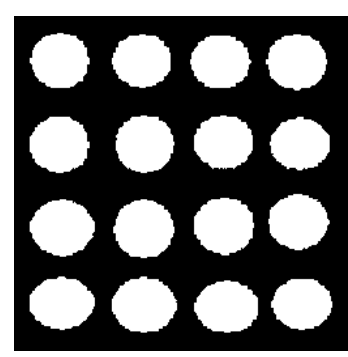

(a)

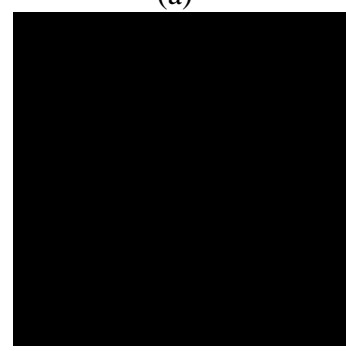

(c)

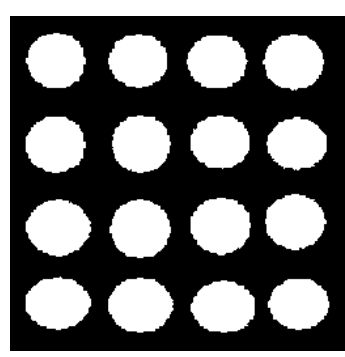

(b)

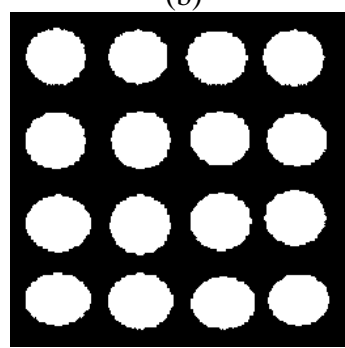

(d)
Fig. 6. Result of circle: (a) Good circle(ITT2); (b) Good circle(ITC); (c) Bad circle(ITT2); (d) Bad circle(ITC)

For the image square, ITT2 obtains the minimal ME value $0.5906 \%$ as shown in Fig.7(a), and the maximal ME value $18.3563 \%$ in Fig.7(c). ITC obtains the minimal ME value $0.6352 \%$ as shown in Fig.7(b), and the maximal ME value 9.3535\% in Fig.7(d). Similar to the image circle, the experimental results prove the above analysis again.

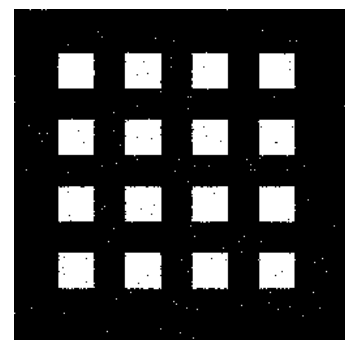

(a)

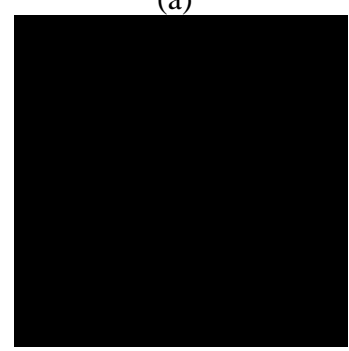

(c)

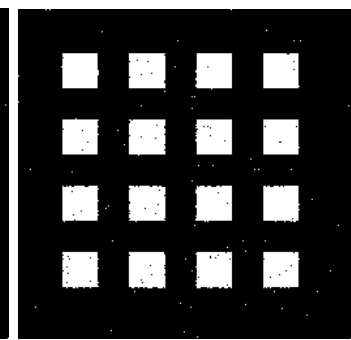

(b)

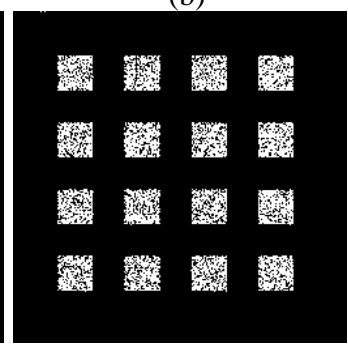

(d)
Fig. 7. Result of square: (a) Good square (ITT2); (b) Good square (ITC); (c) Bad square (ITT2); (d) Bad square (ITC)

Experiment 3: To investigate the randomness of ITC, we provide the third experiment on image thresholding of circle under parameter $\varepsilon=0.0001$. Ten independent experiments (labeled as $\mathrm{E}_{1}-\mathrm{E}_{10}$ ) are conducted with the same parameter. The ME values are calculated as shown in Tab.4. In the ten experiments, the ME values are slightly different. The Standard deviation of ME is 0.00421809 , which is very close to zero, but not equal to zero. The results indicate the segmentation method is random and with a tendency. Furthermore, the mean of ME values is 1.066438 , and there is only a difference about $0.1 \%$ between the mean and the minimal ME value in Tab.1, so it is reasonable that we use the ME value of one experiment to approximate the mean of overall experiments with the same parameter. For simplicity, we use the approach as default.

To observe the random difference by human mind, a transformation is introduced. From Tab.4, we can see the difference between the classified images of $E_{6}$ and $\mathrm{E}_{10}$ is maximal, and then a new image is generated as

$I_{\text {new }}(x, y)=\left\{\begin{array}{ccc}255 & \text { if } & I_{6}(x, y)=I_{10}(x, y) \\ 0 & & \text { otherwise }\end{array}\right.$ 
where $x \in[0, w], y \in[0, h]$ denotes the corresponding horizontal and vertical coordinates of image respectively, $I_{6}(x, y), I_{10}(x, y)$ represents the grayscale value of $(x, y)$ in the sixth and tenth classified image, and $I_{n e w}(x, y)$ is the grayscale value of $(x, y)$ in the new image. The new image is shown as Fig.8, there are several dark points, which is ambitious belong to the object or the background. The existing of these dark pixels indicates ITC is a random method in the probability space. Apparently, the classified image is not severely affected by the difference on the vision in a random way, but more fit the subjective response of human vision, because we feel the scene with a slightly difference at different time in the real world.

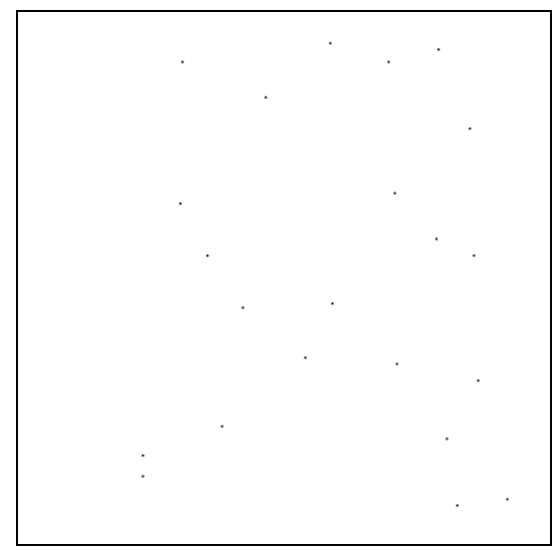

Fig. 8. Randomness of ITC

\subsubsection{Real images}

Experiment 4: To compare the quality of the segmented images, we design the fourth experiment to quantitatively evaluate the overall performance of the selected method against the two traditional popular methods, OTSU ${ }^{31}$ and $\mathrm{FCM}^{32}$. There are 12 real images involved in the experiment, and some of the images select from Berkeley segmentation dataset ${ }^{33}$, and some select from Ref.18. These images contained small and large objects, with clear or fuzzy boundaries. Fig.9 shows these test images and the corresponding groundtruth images. From top left to bottom right, the images are: block, gearwheel, dragonfly, rice, stones, airplane, fluocel, zimba, bird, fruits, cell, and grass.

The ME values of the four methods, which are ITT2, ITC, OTSU, and FCM, are presented in Tab.5, and the minimal ME values denote by bold and italic (0). As is apparent from Tab.5, ITT2 obtains the minimal ME values with four images, ITC obtains five, both are higher than the traditional algorithm (OTSU and FCM). ITT2 and ITC obtain the optimal results with two-thirds of images. For stones, bird, and cell, ITT2 and ITC obtain the suboptimal results, but there are little differences with the optimal results, in which ITC has a difference below $0.5 \%$, and ITT2 has a difference no more than $2 \%$. It suggests that ITT2 and ITC obtain the approximating optimum for all the test images, in some ways, the process introducing the uncertain theory (interval type-2 fuzzy sets or cloud model) into image segmentation is effective.

The statistical result of the ME values is listed in Tab.6. ITT2 and ITC are with average performance $3.5958 \%$ and $3.4783 \%$ respectively, which are clearly inferior to OUST and FCM. The average performance of ITT2 and ITC is only about $60 \%$ of OTSU, and $40 \%$

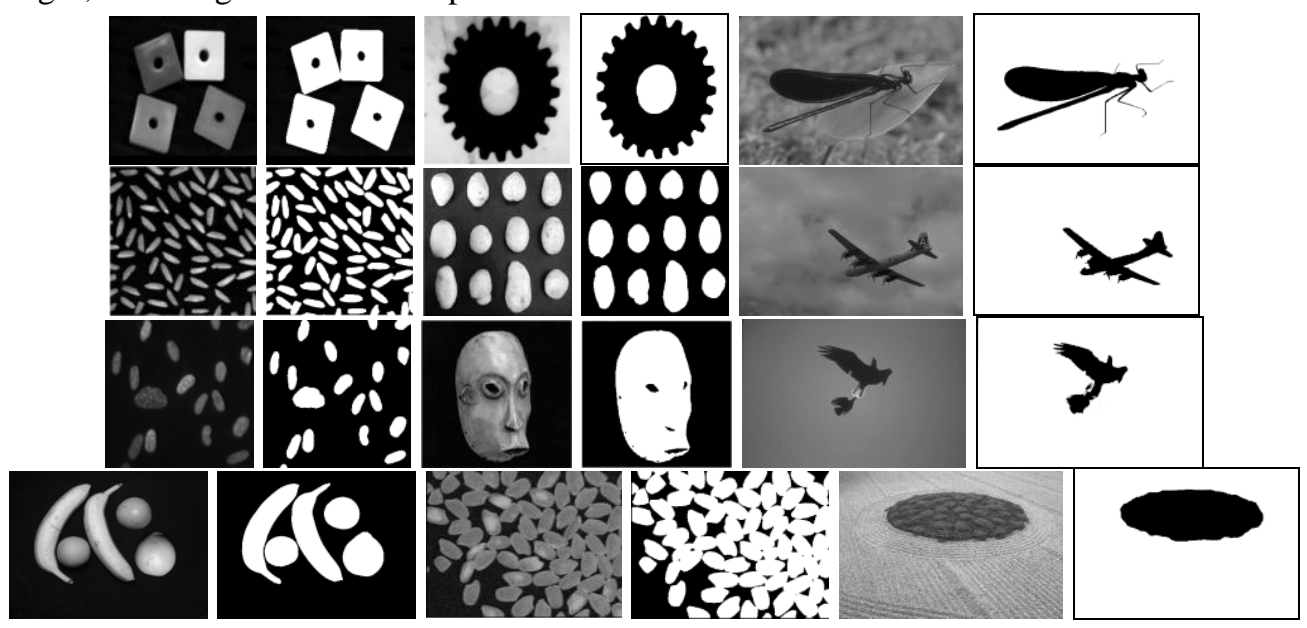

Fig. 9. Test images and the corresponding ground-truth images 
Table 5. Result of experiment 4.

\begin{tabular}{lllllllllllll}
\hline Method & block & fruits & gearwheel & airplane & rice & dragonfly & stones & cell & fluocel & grass & zimba & bird \\
\hline ITT2 & 0.78 & 0.71 & 1.58 & $\mathbf{1 . 4 2}$ & $\mathbf{2 . 2 5}$ & 2.51 & 3.46 & 12.23 & $\mathbf{0 . 4 3}$ & 17.08 & $\mathbf{0 . 1 1}$ & 0.59 \\
ITC & $\mathbf{0 . 1 6}$ & $\mathbf{0 . 0 9}$ & $\mathbf{0 . 4 9}$ & 2.01 & 4.12 & $\mathbf{2 . 2 3}$ & 2.59 & 12.51 & 3.58 & $\mathbf{1 3 . 2 2}$ & 0.26 & 0.48 \\
OTSU & 6.98 & 2.83 & 3.40 & 3.46 & 11.38 & 4.94 & 2.03 & $\mathbf{1 2 . 0 2}$ & 1.06 & 16.52 & 3.10 & $\mathbf{0 . 4 1}$ \\
FCM & 6.79 & 2.98 & 3.40 & 3.75 & 11.38 & 4.65 & $\mathbf{2 . 0 2}$ & 12.02 & 1.24 & 16.52 & 3.34 & 30.22 \\
\hline
\end{tabular}

of FCM. This statistical result reinforces the above conclusion, ITT2 and ITC both can be as the alternative to existing methods.

In sum, the performance of ITC is superior to ITT2, which seem to against the results in section 4.2.1. In fact, these conclusions are consistent with each other, and the reason is that: In theory, ITT2 can obtain the better performance than ITC, but the important prerequisite is that the shape of skeleton membership function and parameter are appropriate for the image, which depends on a careful selection. For the 12 images, we can only try our best to trial the optimal parameters for ITT2, but it can not ensure the parameters are optimal within the limited trials, which limits the application of ITT2 in practice.

Table 6. The statistic of the ME values.

\begin{tabular}{lllll}
\hline & ITT2 & ITC & OTSU & FCM \\
\hline Mean of ME & 3.5958 & 3.4783 & 5.6776 & 8.1925 \\
STD of ME & 5.3567 & 4.5941 & 5.04318 & 8.3869 \\
\hline
\end{tabular}

\subsubsection{Noisy images}

Experiment 5: To investigate the performance of the new algorithm under noisy environment, we conduct the fifth experiment. The circle in Fig.5(a) and the block in Fig. 9 are contaminated by white Gaussian noise, and the mean is 0.2 , standard deviation is 0.01 , as are shown in Figs.10(a)-(b). The fruits and gearwheel in Fig.9 are contaminated by Salt \& Pepper noise, and the noise intensity is 0.05, as are shown in Figs.10(c)-(d).

The ME values of the three methods, which are ITT2, ITC, OTSU, are presented in Tab.7, and the minimal ME values denote by bold and italic (0). As listed in Tab.7, it seems that the three methods respectively have their own merits and deficiencies for a single image. But from the overall performance of four test images, ITC is most effective under such noise conditions, because ITC has the minimal average ME value of $4.7422 \%$ with the lower standard deviation of 1.8287 .

Although ITC has the higher ME values for circle_n and block_n, but the differences with the minimal ME values are 0.1908 and 1.4575 respectively, which is $5 \%$ and $20 \%$ of the minimal ME values. In addition, the two images are contaminated by white Gaussian noise, and one of the theoretical foundations for the universality of normal cloud model is root in the normal distribution, so ITC is more sensitive with Gaussian noise.

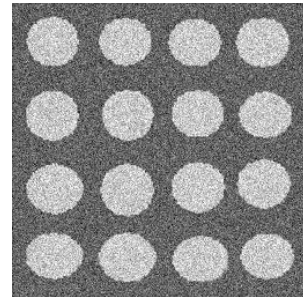

(a)

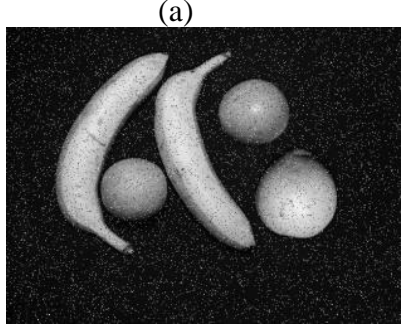

(c)

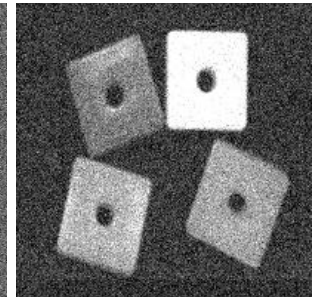

(b)

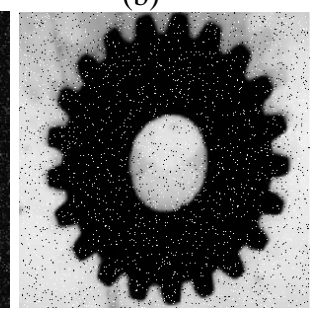

(d)
Fig. 10. The noisy images: (a) circle_n; (b) block_n; (c) fruits_n; (d) gearwheel_n

\section{Discussions and conclusions}

Image thresholding is a difficult task in image processing. Uncertainty is an inherent part in real world applications. The use of new methods for handling incomplete information is of fundamental importance. Interval type- 2 fuzzy sets and normal cloud model both provide the solutions, and they have connections and differences. The paper provides a detail comparison on histogram analysis and thresholing selection. The 
Table 7. Result of experiment 5.

\begin{tabular}{lllllll}
\hline & circle_n & block_n & fruits_n & gearwheel_n & Mean of ME & STD of ME \\
\hline ITT2 & 35.2844 & $\mathbf{5 . 4 5 2 5}$ & 3.1042 & 9.5266 & 13.3419 & 14.8669 \\
ITC & 3.9780 & 6.9100 & $\mathbf{2 . 6 6 8 6}$ & $\mathbf{5 . 4 1 2 4}$ & 4.7422 & 1.8287 \\
OTSU & $\mathbf{3 . 7 8 7 2}$ & 7.2650 & 5.6019 & 5.7819 & 5.6090 & 1.4249 \\
\hline
\end{tabular}

selected methods in the paper, ITT2 and ITC, have showed their versatility and shortcomings.

ITT2 is a supervised method, the shape of skeleton membership function and parameter are the important prerequisite for successful segmentation, and that is, ITT2 uses a unified model (interval type-2 fuzzy set) to represent the histogram along the grayscale, which depends on two crisp functions (LMF and UMF), and the uncertainty analysis is based on an analytic mathematical methods. Therefore, ITT2 is a kind of deductive method and easy to structuring and forming, but how to determinate the shape of skeleton membership function and parameter is too difficult, in addition, there are not enough effective possibility to deal with the randomness in image thresholing. Thus, Specifically defining and calculating a measure of ultrafuzziness will be helpful. As well, methods are necessary to construct type- 2 sets automatically.

While ITC is an unsupervised heuristic method without membership function and parameter, ITC uses probability and mathematical statistics to analyze the uncertainty, and is a kind of inductive method, which heuristically extracts the cloud models from the histogram. So ITC is more robust in theory, and one image segmented by ITC has various results with a slightly difference in a random way, which is more fit the subjective response of human vision in the real world. But ITC is lack of structured formalization, more difficult to interpret, and more sensitive with Gaussian noise.

In image segmentation with uncertainty, even though there are still many challenges to be faced, a successful solution could be devised with a better understanding of the problem and the existing methods. Future research of ITT2, from the extensions point of view, must focus on problems that definition and computation of general type 2 fuzzy sets are tractable ${ }^{10}$. On the other hand, ITC must be more formalized. From the theory basis point of view, type-2 fuzzy sets and cloud model both analyze qualitative concept with uncertainty, there are the relations and the differences between the two uncertain theories ${ }^{16}$. So from the segmentation method point of view, the selected methods are not mutually exclusive, it is more important that how to combine ITT2 and ITC, taking the best features of each.

Since human think with natural languages, exact membership function and crisp set do not exist in human thought, which is easy to be brought into precise kingdom. The methods of probability and mathematical statistics consider randomness. Maybe it is a good way to combine the methods of probability statistics and fuzzy when analyzing the uncertainty in image segmentation. We hope the paper provides a better insight to image segmentation with uncertainty.

\section{Acknowledgements}

The first author is grateful to Dr. Yifu Jin for revising the draft. The authors would like to thank all the anonymous reviewers for their valuable comments and thoughtful suggestions which improved the quality of the presented work. This work was partially supported by the National Natural Science Foundation of China under Grant No.60875007, the National Key Basic Research and Development Program under Grant No.2007CB311003, the Guangdong Natural Science Fund under Grant No.9151027501000039, and the Zhanjiang Science and Technique Plan under Grant No.2009064.

\section{References}

1. Zhang Y.J., Image Segmentation. (Science Press, Beijing, 2001). (in Chinese)

2. Gonzalez, R.C. and R.E. Woods, Digital image processing. 3rd edn. (Prentice Hall, New Jersey, 2008).

3. Sankur, B. and M. Sezgin, Survey over image thresholding techniques and quantitative performance evaluation. J. Electron Imaging. 13(1) (2004) 146-165.

4. Zhang, Y.J., An Overview of Image and Video Segmentation in the Last 40 Years, in Advances in Image and Video Segmentation, eds. Zhang Y.J. (IRM Press: Hershey, 2006), pp. 1-15. 
5. Pantofaru, C. and M. Hebert, A Comparison of Image Segmentation Algorithms (Robotics Institute, Carnegie Mellon University, 2005).

6. Martin, A., H. Laanaya and A. Arnold-Bos, Evaluation for uncertain image classification and segmentation. Pattern Recogn., 39(11)(2006)1987-1995.

7. Martin, A., Fusion for Evaluation of Image Classification in Uncertain Environments, in Proc. 9th International Conference on Information Fusion (IEEE, 2006).

8. Sinha, D. and P. Laplante, A rough set-based approach to handling spatial uncertainty in binary images. Eng. Appl. Artif. Intel., 17(1) (2004) 97-110.

9. Zadeh, L.A., Fuzzy sets. Information and Control, 8(3) (1965)338-353.

10. Bustince, H., et al. , A Survey of Applications of the Extensions of Fuzzy Sets to Image Processing, in BioInspired Hybrid Intelligent Systems for Image Analysis and Pattern Recognition (Studies in Computational Intelligence), eds. P. Melin (Springer, 2009).

11. Zadeh, L.A., The concept of a linguistic variable and its application to approximate reasoning-1. Information Science, 8(3) (1975) 199-249.

12. Mendel, J.M., Type-2 fuzzy sets and systems: an overview. IEEE Computational Intelligence Magazine, 2(1) (2007) 20-29.

13. Li, D.Y. and Du Y., Artificial intelligent with uncertainty (Chapman \& Hall/CRC, Boca Raton, 2007).

14. Li D.Y., Meng H.J., Shi X.M., Membership clouds and membership cloud generators. Computer research and development, 32(6)(1995) 15-20. (in Chinese)

15. Li D.Y., Du Y. Artificial intelligent with uncertainty (National Defence Industry Press, Beijing, 2005).

16. Qin, K., Li D.Y. and Wu T., Comparative Study of Type2 Fuzzy Sets and Cloud Model, in Proc. 5th International Conference on Rough Set and Knowledge Technology (Springer, Beijing, 2010).

17. Bustince, H., et al. , Comment on: Image thresholding using type II fuzzy sets. Importance of this method. Pattern Recogn, 43(9) (2010) 3188-3192.

18. Tizhoosh, H.R., Image thresholding using type II fuzzy sets. Pattern Recogn., 2005. 38(12) (2005) 2363-2372.

19. Tizhoosh, H.R., Type II Fuzzy Image Segmentation, in Fuzzy Sets and Their Extensions: Representation, Aggregation and Models, eds. H. Bustince (Springer, 2008).

20. Vlachos, I.K. and G.D. Sergiadis, Comment on Image thresholding using type II fuzzy sets. Pattern Recogn., 41(5) (2008) 1810-1811.

21. Qin K., Li D.Y., Xu K., Image Segmentation Based on Cloud Model. Journal of Geomatics, 31(5) (2006) 3-5. (in Chinese)

22. Qin K., et al., On the methods of image segmentation with uncertainty, in Proc. 14th International Conference on Geoinformatics (SPIE, 2006).
23. Qin, K., et al. , An image segmentation approach based on histogram analysis utilizing cloud model. in Proc 7th International Conference on Fuzzy Systems and Knowledge Discovery (IEEE, 2010).

24. Mendel, J.M. and R.I.B. John, Type-2 fuzzy sets made simple. IEEE Transaction on Fuzzy Systems, 10(2) (2002) 117-127.

25. Qin K., Novel methods for image segmentation with uncertainty (Wuhan University, Wuhan, 2007). (in Chinese)

26. Li D Y, Liu C Y. Study on the Universality of the Normal Cloud Model. Engineering Sciences, 6(8)(2004) 28-34. (in Chinese)

27. Wang, S., F. Chung and F. Xiong, A novel image thresholding method based on Parzen window estimate. Pattern Recogn., 41(1) (2008) 117-129.

28. Arifina, A.Z. and A. Asano, Image segmentation by histogram thresholding using hierarchical cluster analysis. Pattern Recogn. Lett., 27(13) (2006) 1515-1521.

29. GIMP, 2010-09-10. http://www.gimp.org/

30. Bazi, Y., L. Bruzzone and F. Melgani, Image thresholding based on the EM algorithm and the generalized Gaussian distribution. Pattern Recogn., 40(2) (2007) 619-634.

31. Otsu, N., A threshold selection method from gray-level histograms. IEEE Transactions on Systems, Man, and Cybernetics, 9(1) (1979) 62-66.

32. Bezdek, J.C., Pattern Recognition with Fuzzy Objective Function Algorithms (Kluwer Academic Publishers, Norwell, MA, USA, 1981).

33. Image datasets, U.C. Berkeley, 2009-10-01. http://www.eecs.berkeley.edu/Research/Projects/CS/ 